Voix et Images

voixetimages

\title{
Flamboyants et profonds
}

\section{André Brochu}

Volume 9, numéro 3, printemps 1984

Monique Bosco

URI : https://id.erudit.org/iderudit/200486ar

DOI : https://doi.org/10.7202/200486ar

Aller au sommaire du numéro

\section{Éditeur(s)}

Université du Québec à Montréal

\section{ISSN}

0318-9201 (imprimé)

1705-933X (numérique)

Découvrir la revue

\section{Citer cet article}

Brochu, A. (1984). Flamboyants et profonds. Voix et Images, 9(3), 155-164. https://doi.org/10.7202/200486ar d'utilisation que vous pouvez consulter en ligne.

https://apropos.erudit.org/fr/usagers/politique-dutilisation/ 


\title{
POÉSIE
}

\section{Flamboyants et profonds}

\author{
par André Brochu, Université de Montréal
}

En lisant Une certaine fin de siècle, qui réunit une partie seulement de la production poétique de Claude Beausoleil pendant ces dix dernières années ${ }^{1}$, je réfléchis à ce que fut cette génération qui a produit le mouvement de la Nouvelle Écriture. Au début des années 60, dans l'effervescence de la Révolution tranquille, il y avait eu la prise de parole, par tout un peuple soumis jusque-là aux dictats du clergé et de l'idéologie conservatrice au pouvoir. Cette parole de tous avait trouvé un écho dans la chanson (Vigneault, Léveillée, Ferland...) mais aussi dans le roman, volontiers «joualisant». Le joual en littérature, c'était d'abord et avant tout l'inscription de la volonté de parole collective dans le discours culturel. Quant aux poètes, ils restaient aux antipodes de la parole, à quelques exceptions près (Chamberland, Duguay...).

Dès le début des années 70, un autre mouvement s'amorçait autour de la Barre du jour puis des Herbes rouges, et poussait plus loin la quête du réel. On a cru, poutant, qu'il représentait plutôt la négation du «référent» au profit du seul langage. Et c'est sans doute vrai d'un certain formalisme, mais pas de toute la Nouvelle Ecriture. Ce qui caractérise cette génération, c'est qu'elle met à écrire la même ardeur que tout un peuple mettait à parler, une décennie plus tôt. Pour Beausoleil, pour Charron, pour des Roches et tant d'autres, sans oublier Michel Beaulieu qui fait le lien entre les deux époques, écrire c'est écrire beaucoup, c'est habiter massivement le langage, ce qui nous conduit à cette chose étonnante: un formalisme prolixe. Imagine-t-on Mallarmé publiant, en dix ans, sept cents ou mille pages de vers? Denis Roche lui-même en serait bien incapable. Le formalisme québécois est le contraire d'une poésie «rare», d'une recherche aux confins 
du silence. Il est bien plutôt une énorme promotion du dicible (du scriptible). Chez Beausoleil, en tout cas, il s'agit moins d'annexer de nouveaux territoires que d'occuper, de coloniser ceux déjà conquis, et d'y faire fleurir l'aisance d'une expression lyrique, baroque, très directe aussi. Poésie certainement éloquente car elle se lit - comme elle s'écrit? - au pas de charge. Il faut dire que Beausoleil sait développer un sujet, ne disposant les effets de surprise qu'au niveau de la structure de surface.

Si prendre la parole, en 1960, c'était prendre possession d'un pays, s'emparer de l'écriture, dans les années 70 , s'accompagne d'une reconnaissance de la ville, laquelle suppose d'ailleurs l'acquis du pays mais le dépasse dans deux directions complémentaires: vers l'individualité et le champ de la quotidienneté, d'une part; vers l'universalité concrète d'autre part - celle qui permet d'actualiser en poésie les grands lieux communs de l'existence, sans référence obligée à une situation d'aliénation nationale.

Dès lors, l'écriture peut retrouver la parole; mais celle-ci est devenue la voix illimitée du réel:

\section{j'écris les morceaux d'une conversation infinie le réel alors prend sa place entre les terrasses les livres et les fantasmes}

Trois espaces: la ville, la culture (celle qu'on fait, celle de l'écriture), l'intériorité (l'imaginaire) composent le dispositif hétérogène qui ménage sa place au réel, loin des morosités unidimensionnelles - un réel habitable, chaleureux, où l'humour naît poésie:

... des Amériques à chaque pas

des fêlures et des ombres des rayons qui traversent quand les perles sont des woolworths ...

Impossible de rendre compte de cet immense recueil, où se renouvellent constamment les problématiques du corps, de la fiction, de la ville, de l'écriture, avec parfois des facilités, mais souvent des bonheurs d'expressions considérables, et toujours le même souci: «il faut être flamboyant $/$ il faut ajouter aux matières". Flamboyant, certes, s'oppose à profond, et Beausoleil se perd-facilement dans les subtilités de l'interrogation sur soi (cf. "Un monde en feu»). Il est, en revanche, parfaitement à l'aise dans la fiction quotidienne, qui lui permet d'imaginer que «nous reviendrons comme des Nelligan 1 ... comme des sourires sur ! highway / ... il n'y a plus de résistance / et les fictions planent - śtilles\% («Mémoire de ville», un des plus beaux textes du recueil).

Ajouter aux matières, plutôt qu'aux formes, est bien le fait d'un riç ţe d'Amérique, qui a trouvé dans l'écriture un puissant moyen de vivi , et pour qui la fin de siècle n'a rien, mais absolument rien d'une fin cum monde. 
Dédié «à tous les poètes du pays pour qui la poésie reste un acte de prédilection, de plénitude et de partage», Chant perdu, de Rina Lasnier ${ }^{2}$, ajoute quelques feuillets de beauté pure à une ouvre considérable et dense (bien plus dense que celle de nos modernes formalistes), qui s'étend maintenant sur plus de quarante ans.

Avant tout, prêtons l'oreille d̀ un «chant» qui délaisse la magnificence un peu solennelle de la forme épique, celle des Gisants, de "La Malemer", de «Psyché» ou du Chant de la montée, pour la forme brève, fragmentale qui a toujours côtoyé l'autre et qui tend, de plus en plus, à prendre toute la place. Si la consonne $M$ donne sa tonalité à plusieurs des poèmes épiques (montée, malemer... Marie, qui est au centre de la poétique comme $d u$ poétique chez Rina Lasnier), on dirait que le $P$ organise la forme brève (relisons les quelques mots de la dédicace: poètes, pays, poésie, prédilection, plénitude, partage). $\mathrm{P}$ comme dans Père, comme dans partir - à Jésus qui meurt, le poète dit: "Marie te remet au sein rouvert du Père...» (le poème s'intitule «Partir...»). Et c'est tout le mouvement de la vie, ce don que la matière-mère fait de l'hómme (et de Jésus même) au Père céleste, au terme d'une existence qui a assumé pleinement le mystère immédiat du monde.

Deux traits me frappent dans ces brèves méditations sur la mort, sur les Ecritures et sur la nature qui composent Chant perdu, deux traits qui apparaissent déjà dans les recueils antérieurs et signalent une «manière» qui n'a rien d'une manie. C'est d'abord l'usage abondant des trois points, qui ne signifient ni effusion ni confusion mais ouverture pensive à un au-delà des mots, que les mots extraordinairement suggestifs et précis du poème rendent presque tangible. C'est aussi l'usage du préfixe re-, bien caractéristique de Rina Lasnier:

l'oiseau reboit la pure distance

de la fange au nuage sourcier.

L'acte de reboire est comme le redoublement, du point de vue du Sens, de l'acte naturel précédemment décrit:

\section{L'OISEAU DES GOUTTIËRES}

Je vois d'en bas son regard latéral

quand il boit à la sauvette

à même les pluies placides

décantées à hauteur de gouttières;

(suivent les deux vers cités ci-haut.)

Reboire, c'est l'acte transfiguré par la vérité absolue que le poète fait apparaître au sein des formes quotidiennes; vérité qui ne disqualifie pas les données immédiates du monde, comme l'essence qui s'oppose aux apparences; mais les reprend (re-) et les change en elles-mêmes, dans l'éclairage d'une très douce transcendance. 
Prix Octave-Crémazie 1983, Comme parfois respire la pierre, d'Alain Lessard ${ }^{3}$, est une première ouvre littéraire, conformément aux critères du concours. Le choix du jury semble judicieux car le recueil est d'une sûreté d'écriture et d'une maturité remarquables. On n'y perçoit aucune des complaisances, aucun des flottements d'inspiration d'une plume à ses premiers essais. Sans doute s'agit-il tout de même d'une première ouuvre, où le poète s'applique à établir un registre complet d'effets, de ressources, de motifs; et on ne voit pas toujours la nécessité profonde des propositions poétiques, des arguments qu'il déploie généreusement au cours de ces pages. Néanmoins le tout se termine par une admirable suite («parfois il y a la terre») où le poète joue de tous les procédés mis au point précédemment, en particulier de la répétition qui introduit, au sein d'un langage familier mais soigneusement détourné de ses emplois consacrés, des plages de repos où l'enchantement devient possible.

Comme parfois respire la pierre me semble être un fort bon exemple de ce que pourrait être une poésie post-formaliste, qui congédierait la thématique de l'écriture mais ne rétrograderait pas pour autant vers le discours humaniste (et encore moins nationaliste) qui a précédé. Cette «pierre» qui «respire», elle n'a pas la nostalgie du Sens ou du Centre: elle respire en pierre un air qui est de l'air. On peut parler de poésie du quotidien, car le réel concret immédiat est présent à chaque mot, mais ce n'est pas le quotidien intimiste, couleur de pluie ou couleur chair, de Beaulieu ou de Nepveu, le quotidien-rébus de Laberge ou de Longchamps, le quotidien fantastique de Michel Savard ou de Jocelyne Felx; c'est bien plutôt le quotidien intégral, capable de tout s'assimiler, aussi bien l'isotopie financière («ce placement à vie nous pèse au coeur») que l'isotopie naturelle, culturelle ou sociale:

... si je sue de chaleur dans les carreaux

de ma chemise bien cordée dans vos armoires à mots de vos chèvres laiteuses de livres bien faits notables dans les banlieues vous viendrez dans les armoires leur sucer la lie en complet complet vous seul dans votre automobile bien garée bien garable si huilée de conduite nerveuse mais respectable mais répondant bien

Langage point facile, et qui gagnerait à réduire l'arbitraire de ses impulsions, mais tendu vers une saisie totale, et nouvelle, du vécu.

"Sarzènes»: pierres des alignements mégalithiques (menhirs) en Bretagne, Angleterre, Écosse et Irlande. Vient de «Sarrazin» qui désignait l'étranger. La sarzène, c'est la présence étrangère en plein coeur du pays, et encore ce monument indéchiffrable est-il la signature des ancêtres, de ceux qui sont au plus près des sources collectives. On en conclurait facilement 
que le pays est un malentendu; les origines, un paradoxe. Gérald Godin, qui intitule Sarzènes son dernier recueil de poèmes ${ }^{4}$, s'en garde bien et s'emploie plutôt à renouveler la thématique du pays, en la rendant poreuse au maximum et perméable à tous les courants, tous les souffles de l'ailleurs.

"Sarzènes», déjà, est une image exotique de l'ici puisqu'elle est empruntée à l'Europe. Les poèmes du recueil font souvent penser à Montréal, où les diverses ethnies se côtoient sans se mêler, forment une mosaïque sans dessin ni dessein: «Treblinka Oswiecim Lauzon Coaticook». Un dimanche consacré à lire les «mille pages zebdomadaires / de décrets du conseil du trésor» est baptisé "domenica del tesoro" et, par la grâce de l'exotisme, le terne vécu du politicien accède à cette espèce de fantaisie bien particulière qui, chez Gérald Godin, fonde la poésie.

De la gouaille, de la tendresse, une affection un peu voulue mais réelle pour tous ceux qui composent le pays et en particulier les êtres les plus démunis («ses enfants des parapets / ses guenillous d'autoroutes / ses ramasseux de caps de roues / ses revendeurs de bouteilles vides»); de la lucidité aussi à l'égard des «lobotomisés globaux», des «robotisés que l'on crinque / par tant de longitude / et trop de sollicitudes». Du ressentiment, dans ce poème entièrement en anglais où Godin ressuscite "Octubre» (sic), plaie à jamais ouverte. Et puis un ou deux poèmes d'amour, frais et gratuits comme des mouettes posées sur le mégalithe:

des voiliers de rires passaient dans la chambre et se posaient dans les étangs de ta peau

je tirais dans le tas

chevrotine de baisers

Curieux mélange de rugosité et de délicatesse, qui composent un ton, sinon une écriture poétique soutenue.

C'est avec beaucoup de joie que je salue, dans Aléatoire instantané \& Midsummer $82^{5}$, le retour de Paul Chamberland à une poésie de l'immédiat. Les gros recueils, pleins de wishful thinking et de savoir charlatanesque qui ont marqué la période "nouvelle culture» de notre soldat des temps nouveaux, font place à une sorte de carnet de bord, beaucoup plus modeste. Chamberland retrouve le ton de l'attention aiguê au réel qui caractérisait l'Afficheur hurle ou l'Inavouable. On a l'impression d'une phénoménologie accordée à la profusion de l'actuel - Sartre et Deleuze conjugués:

Tout est en suspension vivant dans le multiple, déliaison/rejonction sans arrêt rendre compte du lieu, de l'occasion le ténu brouillard printanier teintait rose les surfaces qui font l'intersection MontRoyal/St-Urbain ... 
Il n'y a rien, pour autant, d'une régression vers la subjectivité strictement individuelle. Le poète reste l'homme de la collégialité, accordé à l'ensemble du monde (nature et humanité); mais la ferveur «holiste» est plus détendue. À la «volonté de matière autre que celle de l'imagination purement subjective», à la recherche d'un "accomplissement» où le désir et le réel feront enfin bon ménage, se joint le souci de s'en tenir «de plus en plus rigoureusement au réel». Les échos politiques du monde (Beyrouth-Ouest, les Falklands, l'Irak...), les faits divers de toute sorte avoisinent les notations du moment («ce samedi fin mai 82») et du lieu («angle Hutchison/ Villeneuve»), comme si le temps et l'espace quotidiens pouvaient devenir de nouveau, pour la première fois peut-être depuis octobre 1970 , les coordonnées d'événements significatifs.

Il y a bien quelques facilités, comme cet énoncé qui clôt banalement Aléatoire instantané: "le soleil est au bout de chaque rue», ou ce lieu commun qui, malgré la vérité de son assertion, aurait besoin d'être réactivé: «le vingtième siècle aura banalisé le génocide». Mais on est atteint par ce débat du poète avec un multiple qu'il ne cherche plus à harnacher par d'illusoires formules; qu'il accueille simplement,

comme si j'étais devenu un million de reflets.

Tout-ce-qui-arrive me traverse, et $j$ 'en deviens sans fin les facettes tournantes. Je m'égare dans le dédale du multiple. Je fige dans l'affolante modification des apparences. Je n'ai plus de sens

Et quelques images, ou simples dispositions de mots, rappellent le magicien du verbe qui signait Genèse et Terre Québec, en des temps bien différents. Pas forcément meilleurs.

Hugues Corriveau publie Revoir le rouge ${ }^{6}$. J'avais aimé les Taches de naissance ${ }^{7}$ (assez, pas démesurément), pour des raisons semblables à celles qui me font aimer, dans l'ensemble, les brèves proses et le long poème terminal du dernier recueil. Avec, tout de même, des réticences.

C'est de vécu que nous parle Corriveau, un vécu saisi à ras de crue vérité physiologique. Il y a le sexe mais aussi tout le système des humeurs, le grand jeu des pulsions. Et cela parle (est parle), fait des phrases, aligne des sens - sans jamais devenir raison ou passion. Langage très sâr et articulé, en-dessous de la ligne de flottaison qui départage les espaces du corps (de la matière) et de l'esprit; inexistant au-dessus. Voilà du bon matérialisme... En termes kristeviens, on pourrait dire que le symbolique se fait, autant que possible, le redoublement du sémiotique. 
Jamais il n'est question d'amour. Ces poèmes érotiques ignorent le sentiment. La machine du désir' (je me souviens de l'Anti-Oedipe) y est seule exhibée et branchée à d'autres machines: la violence (politique et sociale), l'histoire, le travail, le texte. Il y a, par-ci par-la, un peu de théorie de bonne compagnie, à saveur derridienne; mais surtout des fantasmes de torture, de plaies purulentes, de perversion. Il me semble que le poète en joue de façon plutôt gratuite, et qu'aucune démarche bien nette ne se dégage du recueil.

Je crois, en somme, que Corriveau a un univers bien à lui, et des ressources d'écriture plus que convenables pour le mettre en valeur; mais cet univers reste clos sur lui-même. Il lui manque peut-être cette générosité qui mettrait le langage en circuit avec la vérité pratique de tous. «Revoir le rouge» alors ne serait plus se poser en témoin immobile de la plaie originelle, ou des blessures innombrables du présent; mais retrouver le sens d'une révolution intégrale, sexuelle aussi, d'une promotion du fairehumain. Ce sens - faut-il s'en plaindre? - s'est bien perdu ces derniers temps.

Dans l'Écran, Denise Desautels ${ }^{8}$ fait entendre, avec des habiletés, des calculs, des subterfuges exquis, la voix de l'angoisse. Tout est extrêmement contrölé, propre à créer l'enchantement de la lecture; pourtant tout est poignant, et jamais ne dérangent tant d'adresse et de savoir.

Savoir mythologique: Io, abusée de Zeus, haïe d'Héra, changée en génisse, poursuivie par un taon, affolée, exilée. C'est elle ici qui dit je (en italique, des fragments parlent d'elle a la troisième personne). Elle crie sa folie, son enfance, son impossible rapport aux autres. Ses espoirs furtifs. Ceux de toute femme condamnée, pour une raison ou l'autre, une passion ou l'autre, au silence. Ou au cri. Ou au dangereux corps à corps avec les mots qu'est l'écriture.

Le recours au mythe me rappelle la Nomade, de Julie Stanton", ou plus heureusement le New Medea et le Portrait de Zeus peint par Minerve, sortes de poèmes narratifs de Monique Bosco ${ }^{10}$. Les femmes volontiers universalisent l'expression de leur condition individuelle en la projetant sur une figure transculturelle qui agit un peu comme une image-écran. L'écran: ce qui cache, mais aussi ce qui, bien au contraire, peut servir de support à l'exhibition lumineuse, réfléchir les fantasmes déchaînés.

Savoir de l'écriture: Denise Desautels maîtrise parfaitement ce langage initiatique, cette rhétorique qui sont consubstantiels à la modernité et constituent sans doute un «écran» pour le lecteur ingénu (s'il en est). Mais n'est-ce pas tout langage, même et surtout le plus transparent en apparence, qui cache l'essentiel, se conforte dans l'illusion du «dedans et (du) dehors bien circonscrits»? 
L'histoire d'Io, écrite «à partir de tout et de rien», ressemble à «l'inventaire fabuleux des hauts et des bas d'une ligne droite». Ce motif de la ligne sert en effet de fil conducteur (jusqu'à le devenir expressément), elle est d'abord «la ligne droite claudicante» du vécu quotidien, que brise l'agression étrangère; au passé, elle est «la ligne vigilante» sur laquelle la petite fille «marche droit»; de nouveau dans le présent, c'est le trajet vers l'inconnu qui consiste à mettre «un pied devant l'autre dans l'escalier»; c'est «la ligne de vie prévue à laquelle on déroge un jour», et finalement ce «fil conducteur flexible» qui «ne rompt jamais». Elle symbolise donc avant tout la continuité d'une vie et l'impossibilité d'échapper au destin fixé d'avance, malgré les apparentes indéterminations qu'introduisent les «coups de dés», les agressions.

Présentation graphique particulièrement réussie, par l'auteur luimême (je ne me résigne pas à écrire «auteure»). Elle utilise les caractères Colonial, très ronds, qui font inhabituel, beau et quelque peu gothique: cela distrait d'abord (fait écran), puis séduit. Une angoisse en manteau d'hermine.

Sartre affirmait que le moi est un objet pour la conscience, au même titre que n'importe quel objet du monde. Qu'arrive-t-il quand le moi devient le seul objet, qu'il se substitue au monde et prétend ne faire qu'un avec la conscience? La réponse se trouve peut-être dans Mahler et autres matières, de Pierre Nepveu ${ }^{11}$. Un petit poème, "Mélodrame», pose le problème en ces mots, faussement détachés: «Le moi / n'est pas intéressant. / Ni son bonheur. / Encore moins son malheur. / Le moi est petit / comme une mouche / sur le tablier de maman, / comme un ceuf / brisé par mégarde I sur le plancher de la cuisine.» Parole de l'homme condamné à lui-même, à ressasser un passé plat comme un plancher de cuisine, à ruminer une angoisse boeuf. Cet homme pourrait s'appeler Malheur. Ou Mahler. Que de chutes, de trébuchements, de destructions, d'exécutions, de condamnations, d'effondrements, d'écroulements, de détériorations, de décompositions, de catastrophes, de déracinements, d'effritements, de dérobades, de déchirements, d'éclatements! Et j'en passe. Cet univers est régi par «une physique du moindre espoir», et une logique paradoxale qui se réfère explicitement aux paysages de Max Escher: «Chaque printemps / les idées impérissables / tombaient en poussière.) Heureusement, cet entassement de fins du moi et de fins du monde (les deux se confondent), grâce à l'art qui est grand, devient chant. Prêtant sa voix à Mahler, Nepveu écrit:

Mais ma musique fut intimement liée aux anecdotes, à la couleur du ciel, à la pâte de la terre en forêt quand il pleut, au tremblement du pont sur le fleuve. Elle fut un effondrement de moi-même devant mille choses. Non pas une destruction: plus je m'effondrais, plus j'étais réel. 
La haine de soi, de la chair, de «l'ange pourri de l'avenir», le romantisme exacerbé sont la condition mystique d'une accession à l'autre et à la vie. Ils sont aussi l'envers de la générosité intellectuelle qui caractérise Pierre Nepveu l'universitaire. En fait, on devine cette chaleur de l'homme derrière les thèmes hyperégocentriques du poème. Loin d'être le hochet de l'impudeur, le moi sert de paravent à une extraordinaire passion de l'être, qui s'exprime plus directement sur le plan intellectuel.

L'homosexualité est une dure entreprise. Déviation très naturelle et répandue, en butte aux vexations d'une norme ombrageuse, elle n'en finit pas de lutter pour ses droits et d'affirmer sa différence, comme font toutes les marginalités. André Roy, que ses inclinations sexuelles condamnent à l'obsession, en fait le pain de son écriture, non sans courage mais surtout, avec beaucoup de bonheur, comme en témoignent les Lits de l'Amérique (quatrième volet du Cycle des passions). Je parle du bonheur de l'expression, et de la distance qui transforme l'obsession en poésie. Distance faite d'humour, et sans doute aussi de désespoir neutralisé, d'indifférence conquise de haute lutte. Dans cette distance, qui lui permet de parler de soi à la troisième personne («Si à peine caressés dans le dernier livre / d'André Roy ils se souviennent» etc.), éclôt l'image souvent merveilleuse:

Révérence subtile, à ce moment, de la fumée de cigarette, ses bagues bleues. Je me rappelai que son sperme était un paon souriant, son nombril, trop souriant et grenade grise; $j$ 'ai su alors que le désir, parfois, sanglote comme un fruit, fídele à son sens, à sa géométrie gavée de miel. «J'ai aimé tes couilles roses parce que j'aime les couilles roses.»

On pourra juger étriqué, mais non étrange ou inassimilable, l'univers d'André Roy. Il n'en reste pas moins que si l'amour dit normal a ses chantres depuis la nuit des temps, très peu de poètes ont célébré l'amour prétendu "gai», souvent triste à mourir. Dans cette voie, tout est à inventer, et André Roy s'y emploie avec intelligence et un sens classique de la langue, même quand il se tient dans les franges du lisible.

"cela n'existe pas»: tel est le dernier vers du dernier des poèmes que René Lapierre publie sous le titre de Profil de l'ombre ${ }^{13}$. Il résume bien tout le recueil, en ce sens que le lieu spirituel d'où parle le poète, même si celui-ci s'écrie bravement: «apparences apparences / toute mon âme est à vous», disqualifie le monde. En le transformant justement en apparences, sans cesse opposées à l'éternité, l'absolu, l'infini dissolvants. Dieu seul, ou un ange, peut concevoir la formidable paronomase du deuxième vers, dans ce «Portrait à la fenêtre»: 
ici ou là

ames ou amibes

difficile de distinguer

difficile de voir ...

Âmes ou amibes, qu'importe?! Il y a le poète, ce qui l'entoure, celle qu'il aime (ou qu'il attend), et le sentiment malgré tout accablant de la solitude et de la mort. Les humains sont des «lunes d'argile», des «reflets» de l'astre «au fond du puits». Ils attendent d'être réunis à la source de l'être. Tous les objets de ce monde sont «choses de rien / nuit absolue». Les hommes sont des «mortels / à qui manque la mort».

On a compris que la poésie de René Lapierre est métaphysique et, comme toute métaphysique, désespérée. Son pessimisme rappelle celui de Nepveu, même si les poétiques diffèrent. Il a plus d'émotion chez ce dernier, qui affectionne l'élégie quotidienne; plus de sobriété chez Lapierre. Les images sont rares car, dans un univers où chaque objet est cerné de néant, perdu de solitude, rien n'avalise les connexions métaphoriques. Ce n'est plus Mahler mais Webern, si l'on veut. C'est parfois aussi Saint-Denys Garneau: «nous entendons / quelquefois nos fantômes s'agiter / avec des cris d'oiseaux». C'est surtout René Lapierre, auteur d'un excellent petit recueil, qui, à la semblance du Phénix kostrowitskien,

... s'il meurt un soir

Le matin voit sa renaissance.

1. Claude Beausoleil, Une certaine fin de siècle, Saint-Lambert, Noroît, 1983, $350 \mathrm{p}$.

2. Rina Lasnier, Chant perdu, Trois-Rivières, Ecrits des Forges, 1983, 98 p.

3. Alain Lessard, Comme parfois respire la pierre, Montréal, Leméac, 1983, 92 p.

4. Gérald Godin, Sarzènes, Trois-Rivières, Ecrits des Forges, 1982, 56 p.

5. Paul Chamberland, Aléatoire instantané \& Midsummer 82, Trois-Rivières, Ecrits des Forges, 1983, 72 p.

6. Hugues Corriveau, Revoir le rouge, Montréal, VLB éditeur, 1983, 154 p.

7. Hugues Corriveau, les Taches de naissance, Montréal, les Herbes rouges, $\mathrm{n}^{\circ} 101$, mars $1982,30 \mathrm{p}$.

8. Denise Desautels, l'Écran, Saint-Lambert, Noroit, 1983, 90 p.

9. Julie Stanton, la Nomade, Montréal, l'Hexagone, 1982, 55 p.

10. Monique Bosco, New Medea, Montréal, l'Actuelle, 1974, 150 p.; Portrait de Zeus peint par Minerve, Montréal, HMH, $1982,180 \mathrm{p}$.

11. Pierre Nepveu, Mahler et autres matieres, Saint-Lambert, Noroît, 1983, 76 p.

12. André Roy, les Lits de l'Amérique, Montréal, les Herbes rouges, nos 116-117, $1983,62 \mathrm{p}$.

13. René Lapierre, Profil de l'ombre, Trois-Rivières, Ecrits des Forges, 1983, 80 p. 